\title{
UPPER SEMICONTINUITY OF ATTRACTORS FOR SMALL RANDOM PERTURBATIONS OF DYNAMICAL SYSTEMS
}

\author{
${ }^{1}$ Tomás Caraballo, ${ }^{1}$ José A. Langa and ${ }^{2,3}$ James C. Robinson \\ ${ }^{1}$ Departamento de Ecuaciones Diferenciales y Análisis Numérico \\ Universidad de Sevilla. Apdo. Correos 1160 \\ 41080-Sevilla. Spain \\ e-mails: caraballo@numer.us.es, langa@numer.us.es \\ ${ }^{2}$ Department of Applied Mathematics and Theoretical Physics \\ University of Cambridge. Silver Street, CB3 9EW \\ Cambridge. U.K. \\ ${ }^{3}$ New address: Oxford University, Centre for Industrial and Applied Mathematics, \\ Mathematical Institute, 24-29 St Giles, Oxford OX1 3LB. U.K. \\ e-mail: robinso4@maths.ox.ac.uk
}

December 2, 2001

\begin{abstract}
The relationship between random attractors and global attractors for dynamical systems is studied. If a partial differential equation is perturbed by an $\epsilon$-small random term and certain hypotheses are satisfied, the upper semicontinuity of the random attractors is obtained as $\epsilon$ goes to zero. The results are applied to the Navier-Stokes equations and a problem of reaction-diffusion type, both perturbed by an additive white noise.
\end{abstract}

This paper has appeared in Communications in PDEs 23 (1998) 1557-1581 


\section{Introduction}

The asymptotic behaviour of dynamical systems is one of the most important problems of modern mathematical physics and the theory has been greatly developed over the last decade or so. In the deterministic case the global attractor, a compact invariant and attracting set, occupies a central position (see, for example, Hale [10] and Temam [21]).

In fact, one of the most important discoveries has been the finite-dimensionality of the attractors in mathematical models for fluid dynamics. However, much less developed is the analogous subject when random perturbations appear in the systems under investigation. In order to overcome this problem some authors have introduced a different notion of an attractor for stochastic partial differential equations (for example, Morimoto [16] and Schmalfuß [19] have worked with the concept of probability attractors).

Recently, Crauel and Flandoli [5] (see also Schmalfuß [18] and Crauel et al. [4]) have introduced a more geometrical attractor (defined again as a set in the phase space) for some random dynamical systems, as a generalisation of the classical concept of the global attractor for many models in Physics, Chemistry and Biology. The result we present in this work gives some indication that their definition is a good generalisation of the deterministic concept.

When a partial differential equation modelling a dynamical system is perturbed by, for example, an additive white noise, we get a non-autonomous system, but one for which the trajectories have very large fluctuations as $t$ goes to infinity. This is why the theory of random attractors (in the sense of Crauel and Flandoli [5]) does not follow the theory of global attractors for non-autonomous systems (see Sell [20], Vishik [22], Haraux [13]), where some properties of compactness on the non-autonomous term are needed. Instead, the random attractor is defined as a random invariant compact set, attracting all the bounded deterministic sets from " $t=-\infty$ ". Furthermore, due to the large fluctuations of the trajectories when time evolves, it is possible that the probability that the random attractor has nonempty intersection with $U$ is positive, for any open $U$.

The main question we study in this paper is the relationship between the random attractor and the deterministic one when we apply to our (deterministic) partial differential equation a small random perturbation, whose strength is measured by a parameter $\epsilon$. Recently, Kloeden and Stoiner [14] have obtained some results on the relation between the attractors for an autonomous ordinary differential equation and small non-autonomous perturbations. What we expect in our case, and are able to prove under some conditions, is that 
the random attractor is a random perturbation of the deterministic one in the sense that, given a $\delta>0$, with probability one there exists $\varepsilon$ (depending on $\omega$, a particular event in a probability space $(\Omega, \mathcal{F}, P)$ ) sufficiently small, such that the random attractor is inside the $\delta$-neighbourhood of the global attractor for all $\epsilon \leq \varepsilon$. In fact, we prove a theorem on upper semicontinuity for random attractors. Roughly speaking, if $\mathcal{A}_{\epsilon}$ is the attractor associated to the perturbed dynamical system and $\mathcal{A}_{0}$ corresponds to the unperturbed one, we say that these attractors have the property of upper semicontinuity if

$$
\lim _{\epsilon \rightarrow 0} \operatorname{dist}\left(\mathcal{A}_{\epsilon}, \mathcal{A}_{0}\right)=0,
$$

where dist(., .) is the Hausdorff semidistance $\operatorname{dist}(A, B)=\sup _{a \in A} \inf _{b \in B} d(a, b)$, on a metric space $(X, d)$. Note that this is different from the Hausdorff metric, which is defined as

$$
\sup \{\operatorname{dist}(A, B), \operatorname{dist}(B, A)\} .
$$

There are some results of this type in the deterministic case (see Hale [10], Hale et al. [11], Hale and Raugel [12], Temam [21] among others). Most of them need some properties of uniform convergence on bounded sets of time and space of the perturbed semigroup to the unperturbed one and the existence of a uniform absorbing set for all the semigroups. For the random perturbation of a dynamical system which appears in this paper we prove a similar result with probability one.

In section 2, we present the main result of this paper, that is, a theorem on the upper semicontinuity on random attractors. We apply this result to the Navier-Stokes equations and a reaction-diffusion problem with additive noise in sections 3 and 4 respectively. In the last section some conclusions and possible generalizations are discussed.

\section{Upper semicontinuity of random attractors}

Firstly, let us introduce the framework in which the theory of random attractors is developed (see Crauel and Flandoli [5] for more details).

Let $(\Omega, \mathcal{F}, P)$ be a probability space and $\left\{\theta_{t}: \Omega \rightarrow \Omega, t \in \mathbb{R}\right\}$ a family of measure preserving transformations such that $(t, \omega) \mapsto \theta_{t} \omega$ is measurable, $\theta_{0}=i d, \theta_{t+s}=\theta_{t} \theta_{s}, \forall s, t \in \mathbb{R}$. The flow $\theta_{t}$ together with the probability space, $\left(\Omega, \mathcal{F}, P,\left(\theta_{t}\right)_{t \in \mathbb{R}}\right)$ is called a (measurable) dynamical system. 
A random dynamical system (RDS) on a Polish space $(X, d)$ with Borel $\sigma$-algebra $\mathcal{B}$ over $\theta_{t}$ on $(\Omega, \mathcal{F}, P)$ is a measurable map

$$
\begin{aligned}
\varphi: \mathbb{R}^{+} \times \Omega \times X & \rightarrow X \\
(t, \omega, x) & \mapsto \varphi(t, \omega) x
\end{aligned}
$$

such that

i) $\varphi(0, \omega)=i d \quad($ on $X)$

ii) $\varphi(t+s, \omega)=\varphi\left(t, \theta_{s} \omega\right) \circ \varphi(s, \omega), \forall t, s \in \mathbb{R}^{+}$, for almost all $\omega \in \Omega$ (cocycle property).

A RDS is continuous or differentiable if $\varphi(t, \omega): X \rightarrow X$ is continuous or differentiable (see Arnold and Crauel [1] for more details on RDS).

A random set $K(\omega) \subset X$ is said to absorb the set $B$ if for almost all $\omega \in \Omega$ there exists $t(B, \omega)$ such that $\forall t \geq t(B, \omega)$

$$
\varphi\left(t, \theta_{-t} \omega\right) B \subset K(\omega) .
$$

Finally, a random set $\mathcal{A}(\omega)$ is a random attractor associated to the $\operatorname{RDS} \varphi$ if $P$-almost surely $(P-$ a.s., for short $)$

i) $\mathcal{A}(\omega)$ is a random compact set, namely, $\mathcal{A}: \Omega \rightarrow 2^{X}$ is a set valued map taking values in the compact subsets of $X$ and being measurable, i.e., for each $x \in X$ the map $\omega \in \Omega \mapsto \operatorname{dist}(\{x\}, \mathcal{A}(\omega))$ is measurable.

ii) $\varphi(t, \omega) \mathcal{A}(\omega)=\mathcal{A}\left(\theta_{t} \omega\right), \forall t \geq 0 \quad$ (invariance) and

iii) for all $B \subset X$ bounded (and non-random)

$$
\lim _{t \rightarrow \infty} \operatorname{dist}\left(\varphi\left(t, \theta_{-t} \omega\right) B, \mathcal{A}(\omega)\right)=0 .
$$

Remark. Note that the random attractor $\mathcal{A}(\omega)$ attracts every bounded set "from $-\infty$ ", as given $u_{0} \in B, \varphi\left(t, \theta_{-t} \omega\right) u_{0}$ represents the position at time $t=0$ of the trajectory which was in $u_{0}$ at time $-t$.

In this situation, we have the following theorem of existence of random attractors due to Crauel and Flandoli ([5], theorem 3.11).

Theorem 1 Suppose that there exists a random compact set $K(\omega)$ absorbing every bounded non-random set $B \subset X$. Then, the set

$$
\mathcal{A}(\omega)=\overline{\bigcup_{B \subset X} \Lambda(B, \omega)}
$$


is a random attractor for $\varphi$, where the union is taken over all $B \subset X$ bounded, and $\Lambda(B, \omega)$ is the omega-limit set of $B$ and is given by

$$
\Lambda(B, \omega)=\bigcap_{T \geq 0} \overline{\bigcup_{t \geq T} \varphi\left(t, \theta_{-t} \omega\right) B} .
$$

Furthermore, we know that the random attractor is the smallest random set satisfying iii) and the largest invariant (in the sense of ii)) set (see Crauel et al. [4]). Finally, in Crauel [3] it is shown that random attractors are unique.

All these results suggest that, although in general the random attractor is not a uniformly bounded set, this concept is a good generalisation of that of global attractor. In some cases, random attractors appear by a random perturbation of a dissipative partial differential equation, so that a result on the upper semicontinuity of random attractors to the global attractor would help to confirm that this is the right extension of the concept to the field of random dynamical systems.

Suppose now we have a (deterministic) dynamical system on a Polish space $(X, d), S(t): X \rightarrow X, t \in \mathbb{R}^{+}$and that there exists a global attractor $\mathcal{A}$ for $S(t)$ given as $\Lambda(U)$, the omega limit set of $U$, for some bounded absorbing set $U$. Various conditions for the existence of such a global attractor $\mathcal{A}$ are given in the books of Hale [10] (Chapter 3, p.39), and Temam [21] (Chapter I, Theorem 1.1, p.23)), the simplest being that the absorbing set $U$ is compact.

Let us perturb our dynamical system by the addition of a random element depending on a parameter $\epsilon \in(0,1]$, so that we obtain a random dynamical system

$$
\varphi_{\epsilon}: \mathbb{R}^{+} \times \Omega \times X \rightarrow X
$$

such that for $P$-almost every $\omega \in \Omega$ and all $t \in \mathbb{R}^{+}$

$$
\varphi_{\epsilon}\left(t, \theta_{-t} \omega\right) x \rightarrow \mathcal{S}(t) x \text { as } \epsilon \searrow 0
$$

uniformly on bounded sets of $X$.

Then, we can prove the following result on the relation of the attractors associated to these systems:

Theorem 2 Assume that for all $\epsilon \in(0,1]$ there exists a random attractor $\mathcal{A}_{\epsilon}(\omega)$, and that in addition there exists a compact set $K$ such that, $P-$ a.s.

$$
\lim _{\epsilon \backslash 0} \operatorname{dist}\left(\mathcal{A}_{\epsilon}(\omega), K\right)=0
$$


Then,

$$
\lim _{\epsilon \rightarrow 0} \operatorname{dist}\left(\mathcal{A}_{\epsilon}(\omega), \mathcal{A}\right)=0 \text { with probability one. }
$$

Furthermore, if for $\epsilon_{0} \in(0,1]$ we have that for $P$-almost every $\omega \in \Omega$ and all $t \in \mathbb{R}^{+}$

$$
\varphi_{\epsilon}\left(t, \theta_{-t} \omega\right) x \rightarrow \varphi_{\epsilon_{0}}\left(t, \theta_{-t} \omega\right) x \quad \text { as } \epsilon \rightarrow \epsilon_{0}
$$

uniformly on bounded sets of $X$, then the convergence above is upper semicontinuous in $\epsilon$, that is

$$
\lim _{\epsilon \rightarrow \epsilon_{0}} \operatorname{dist}\left(\mathcal{A}_{\epsilon}(\omega), \mathcal{A}_{\epsilon_{0}}(\omega)\right)=0 \quad \text { with probability one. }
$$

Remark. Note that, given (H1), (H2) is in fact a necessary and sufficient condition for the upper semicontinuity property. Sufficiency is the content of the theorem, and necessity follows since upper-semicontinuity is precisely (H2) with $K=\mathcal{A}$, a compact set.

In applications, (H2) will follow from a similar property for the random absorbing sets which are in fact used to obtain the random attractors:

Lemma 1 Suppose that there exists a family of random compact absorbing (uniformly in $\epsilon$ ) sets $K_{\epsilon}(\omega)$, that is, for $P$-almost every $\omega \in \Omega$ and all $B \subset X$, there exists $t_{B}(\omega)$, independent of $\epsilon$ such that

$$
\forall t \geq t_{B}(\omega), \quad \varphi_{\epsilon}\left(t, \theta_{-t} \omega\right) B \subset K_{\epsilon}(\omega), \quad \forall \epsilon \in\left(0, \epsilon_{0}\right]
$$

and that there exists a compact set $K$ such that $P-a . s$.

$$
\lim _{\epsilon \backslash 0} \operatorname{dist}\left(K_{\epsilon}(\omega), K\right)=0
$$

Then for each $\epsilon \in(0,1]$ there exists a random attractor $\mathcal{A}_{\epsilon}(\omega)$, and $(\mathrm{H} 2)$ holds. 
Such a property is a consequence of the fact that the random perturbation to the system vanishes as $\epsilon$ tends to zero. However, in applications we have to follow all the computations carefully in order to verify this condition with probability one.

In most of the examples we can also ensure that the shift $\theta_{t}$ is ergodic. Crauel et al. [4] have proved in this situation that there exists a bounded set $B \subset X$ such that the random attractor is the omega-limit set of $B$ with probability one. Moreover, in some of the applications (see section 4) we can get that the random attractor is a subset of a random absorbing ball $B(x, r(\omega)) \subset X$ (for a suitable $x \in X$ ) such that $r(\omega)$ has finite expectation. The following result generalizes that in [4] under the hypothesis of finite expectation of the radius of the absorbing ball, showing how all the $\epsilon$-dependent random attractors can be expressed as the omega limit set of a fixed bounded and non-random ball in $X$ :

Proposition 1 Assume that $\theta_{t}$ is ergodic, and that there exists a random absorbing ball $B\left(x, r_{\epsilon}(\omega)\right)$ (for a fixed $x \in X$ ) on $X$ for $\varphi_{\epsilon}$ such that $P$-a.s.

$$
\sup _{\epsilon \in(0,1]} r_{\epsilon}(\omega)<\infty
$$

and there exists $p \geq 1$ with

$$
E\left(\sup _{\epsilon \in(0,1]}\left|r_{\epsilon}(\omega)\right|^{p}\right)<+\infty .
$$

Then, there exists a ball $B_{R}=B(x, R) \subset X$ such that $P-$ a.s.

$$
\mathcal{A}_{\epsilon}(\omega)=\Lambda_{\epsilon}\left(B_{R}, \omega\right) \quad \forall \epsilon \in(0,1]
$$

where $\Lambda_{\epsilon}$ denotes the omega-limit set for the random dynamical system $\varphi_{\varepsilon}$.

\subsection{Proof of the results}

For the proof of theorem 2 we will need the following lemma (cf. Ladyzhenskaya $[15])$ 
Lemma 2 Suppose we have a decreasing family of compact sets $\left\{L_{n}\right\}_{n \in N}$ in a metric space $(X, d)$ and $\delta_{n} \rightarrow 0,\left\{x_{n}\right\}_{n \in N}$ such that

$$
\operatorname{dist}\left(x_{n}, L_{n}\right) \leq \delta_{n}
$$

Then there exists a subsequence $x_{n_{j}}$ converging to $x \in \bigcap_{n \in N} L_{n}$.

Proof of theorem 2. Let us take $\epsilon_{j} \searrow 0$ as $j \rightarrow \infty$ and $x_{j} \in \mathcal{A}_{\epsilon_{j}}(\omega)$ such that $x_{j} \rightarrow x_{0}$. Then the theorem is proved if we show that $x_{0} \in \mathcal{A}=\Lambda(K)$. Indeed, suppose that the result is not true; then there exists $\epsilon_{j} \searrow 0$ as $j \rightarrow \infty$ and $\delta>0$ such that

$$
\operatorname{dist}\left(\mathcal{A}_{\epsilon_{j}}(\omega), \mathcal{A}\right)>\delta, \quad \forall j \in \mathbb{N}
$$

and thus there exists a sequence $\left\{x_{j}\right\} \in \mathcal{A}_{\epsilon_{j}}(\omega)$ such that, for all $x \in \mathcal{A}$,

$$
d\left(x_{j}, x\right)>\delta \quad \forall j \in \mathbb{N} .
$$

We thus obtain a contradiction if it is possible to find a subsequence of the $x_{j}$ which is convergent. But this is guaranteed by (H2) along with lemma 2, since $x_{j} \in \mathcal{A}_{\epsilon_{j}}(\omega)$.

Due to the invariance of the random attractor, we can write that, for all $n \in \mathbb{N}$,

$$
x_{j}=\varphi_{\epsilon_{j}}\left(n, \theta_{-n} \omega\right) y_{j}^{n},
$$

with $y_{j}^{n} \in \mathcal{A}_{\epsilon_{j}}\left(\theta_{-n} \omega\right)$, but we know that

$$
\lim _{j \rightarrow \infty} \operatorname{dist}\left(\mathcal{A}_{\epsilon_{j}}\left(\theta_{-n} \omega\right), K\right)=0,
$$

so that

$$
\lim _{j \rightarrow \infty} d\left(y_{j}^{n}, K\right)=0 .
$$

Thus, we have a compact set $K$ and we can take a sequence $\delta_{j} \rightarrow 0$ such that

$$
d\left(y_{j}^{n}, K\right) \leq \delta_{j} .
$$

Using lemma 2 again, there exists a subsequence (that we relabel again as $y_{j}^{n}$ ) which converges to a point $y_{0}^{n} \in K$. So we have

$$
\lim _{j \rightarrow \infty} x_{j}=x_{0}=\lim _{j \rightarrow \infty} \varphi_{\epsilon_{j}}\left(n, \theta_{-n} \omega\right) y_{j}^{n} .
$$


But note that, $P-$ a.s., $\varphi_{\epsilon_{j}}\left(n, \theta_{-n} \omega\right) y_{j}^{n}$ converges to $\mathcal{S}(n) y_{0}^{n}$, since

$$
\begin{aligned}
& \left|\varphi_{\epsilon_{j}}\left(n, \theta_{-n} \omega\right) y_{j}^{n}-\mathcal{S}(n) y_{0}^{n}\right| \\
& \leq\left|\varphi_{\epsilon_{j}}\left(n, \theta_{-n} \omega\right) y_{j}^{n}-\mathcal{S}(n) y_{j}^{n}\right|+\left|\mathcal{S}(n) y_{j}^{n}-\mathcal{S}(n) y_{0}^{n}\right|
\end{aligned}
$$

and, by (H1), as $\left\{y_{j}^{n}\right\}$ is bounded, for all $\delta>0 \exists j_{0}$ such that for all $j \geq j_{0}$

$$
\left|\varphi_{\epsilon_{j}}\left(n, \theta_{-n} \omega\right) y_{j}^{n}-\mathcal{S}(n) y_{j}^{n}\right| \leq \delta / 2
$$

and, by continuity of $\mathcal{S}(n), \exists j_{1}$ such that $\forall j \geq j_{1}$

$$
\left|\mathcal{S}(n) y_{j}^{n}-\mathcal{S}(n) y_{0}^{n}\right| \leq \delta / 2
$$

and this implies that there exists a subsequence (relabeled again as $y_{j}^{n}$ ) which converges $P-a . s$., so that we can assure that

$$
x_{0}=\mathcal{S}(n) y_{0}^{n},
$$

with $y_{0}^{n} \in K$, for all $n \in \mathbb{N}$. Since $U$ is an absorbing set, there exists some $t_{0}(K)$ such that

$$
S(t) K \subset U \quad \forall t \geq t_{0}(K),
$$

and so we can write, for all $n \geq t_{0}(K)$,

$$
x_{0}=\mathcal{S}\left(n-t_{0}(K)\right)\left[\mathcal{S}\left(t_{0}(K)\right) y_{0}^{n}\right]=\mathcal{S}\left(n-t_{0}(K)\right) z_{0}^{n},
$$

with $z_{0}^{n} \in U$. Since $\mathcal{A}=\Lambda(U)$, it is clear that $x_{0} \in \mathcal{A}$, which finishes the proof of ii).

Note that, by (H3), every occurrence in the proof of $\mathcal{S}$ could have been replaced with $\varphi_{\epsilon_{0}}$ for some $\epsilon_{0} \in(0,1]$, yielding a proof of the upper semicontinuity in $\epsilon$ of the random attractors.

Proof of lemma 1. The existence of the random attractors $\mathcal{A}_{\epsilon}(\omega)$ for each $\epsilon \in(0,1]$ follows from theorem 1 . It thus only remains to show that

$$
\lim _{\epsilon \searrow 0} \operatorname{dist}\left(\mathcal{A}_{\epsilon}(\omega), K\right)=0 .
$$

Indeed, since $K_{\epsilon}(\omega)$ is uniformly absorbing, given $B \subset X$ bounded, it is easy to see that $\Lambda_{\epsilon}(B, \omega) \subset K_{\epsilon}(\omega)$ and then, $P-$ a.s.,

$$
\mathcal{A}_{\epsilon}(\omega) \subset K_{\epsilon}(\omega)
$$


which implies, from $\left(\mathrm{H} 2^{\prime}\right)$, that $P-$ a.s.

$$
\lim _{\epsilon \backslash 0} \operatorname{dist}\left(\mathcal{A}_{\epsilon}(\omega), K\right)=0 .
$$

which is $(\mathrm{H} 2)$.

Proof of proposition 1. We know that, with probability one

$$
\mathcal{A}_{\epsilon}(\omega) \subset B\left(x, r_{\epsilon}(\omega)\right) .
$$

Furthermore, there exists $p \geq 1$ such that

$$
E\left(\sup _{\epsilon \in(0,1]}\left|r_{\epsilon}(\omega)\right|^{p}\right)=M_{p}<+\infty,
$$

and so, given $R>0$, by Tchebychev's inequality,

$$
P\left(\sup _{\epsilon \in(0,1]}\left|r_{\epsilon}(\omega)\right|>R\right) \leq \frac{M_{p}}{R^{p}}
$$

from which we can deduce

$$
P\left(\sup _{\epsilon \in(0,1]}\left|r_{\epsilon}(\omega)\right| \leq R\right)>1-\frac{M_{p}}{R^{p}} .
$$

Then, it is clear that choosing $R>\left(M_{p}\right)^{1 / p}$, and taking into account that $P\left(\mathcal{A}_{\epsilon}(\omega) \subset B\left(x, \sup _{\epsilon \in(0,1]} r_{\epsilon}(\omega)\right)\right)=1$ we get

$$
P\left(\mathcal{A}_{\epsilon}(\omega) \subset B(x, R)\right)>0 .
$$

Now we need the following result in Crauel [3]:

Proposition 2 Suppose that $\varphi$ is a random dynamical system and $\mathcal{A}(\omega)$ is a random attractor associated to $\varphi$. Then, if the flow $\theta_{t}$ is ergodic,

$$
P(\mathcal{A}(\omega)=\Lambda(D, \omega))=1
$$

for every non-random $D \subset X$ with $P(\mathcal{A}(\omega) \subset D)>0$.

In our situation we have that, for all $\epsilon \in(0,1]$

$$
P\left(\mathcal{A}_{\epsilon}(\omega) \subset B(x, R)\right)>0,
$$

and consequently

$$
P\left(\mathcal{A}_{\epsilon}(\omega)=\Lambda_{\epsilon}(B(x, R), \omega)\right)=1, \quad \forall \epsilon \in(0,1] .
$$




\section{Small random perturbation of Navier-Stokes equations by additive white noise}

One of the most interesting examples where the theory of random attractors has been applied is the Navier-Stokes equations with additive noise (see Crauel and Flandoli [5] and Crauel et al. [4]). We prove that the hypotheses of lemma 1 are satisfied and so theorem 2 is applicable and we obtain the upper semicontinuity of the random attractors as $\epsilon \rightarrow 0$.

\subsection{Formulation of the problem}

We will now give the abstract framework for the Navier-Stokes equations in a two-dimensional domain $\Omega$,

$$
\frac{\partial u}{\partial t}-\nu \Delta u+(u \cdot \nabla) u+\nabla p=f \quad \nabla \cdot u=\left.0 \quad u\right|_{\partial \Omega}=0,
$$

perturbed by an additional additive white noise. Note that we restrict attention to the two-dimensional equation since, even in the deterministic case, existence and uniqueness questions for three-dimensional domains remain unresolved (see [7] for example).

Let $H$ be a separable Hilbert space (inner product $(.,$.$) and norm |.|) and$ $A: D(A) \subset H \rightarrow H$ a self-adjoint strictly positive linear operator in $H$. Let $V=D\left(A^{1 / 2}\right)$ be a separable Hilbert space (inner product $((.,)$.$) and norm \|$.$\| )$ which is compactly embedded in $H$ if we also assume that $A^{-1}$ is compact. Then we have that, if $\lambda_{1}$ is the first eigenvalue of $A$,

$$
\|u\|^{2} \geq \lambda_{1}|u|^{2}, \quad \forall u \in V .
$$

Let $B(u, v)$ be a bilinear continuous operator from $V \times V \rightarrow V^{\prime}$ ( $V^{\prime}$ is the dual space of $V$ ) and suppose that there exists $c_{0}$ such that

$$
\mid(B(u, v), z))\left.\left|\leq c_{0}\right| u\right|^{1 / 2}|| u||^{1 / 2}|v|^{1 / 2}\|v\|^{1 / 2}|| z \|
$$

for all $u, v, z \in V$ and

$$
(B(u, v), v)=0
$$

for all $u, v \in V$.

Given $f \in H$ and $\phi \in D(A)$, we consider a small random perturbation of the 
abstract deterministic Navier-Stokes equation given by

$$
d u_{\epsilon}+A u_{\epsilon} d t+B\left(u_{\epsilon}, u_{\epsilon}\right) d t=f d t+\epsilon \phi d W(t), \quad u_{\epsilon}(0)=u_{0},
$$

where $W(t)$ is a real valued two-sided Wiener process and such that there exists $c_{1}>0$ for which

$$
|(B(u, \phi), u)| \leq c_{1}|u|^{2}, \quad \forall u \in V
$$

Remark. The analysis can be extended to the case $\epsilon \sum_{i=1}^{d} \phi^{i} d W_{t}^{i}$, but we work on (4) for clarity in the exposition.

It is known (see Temam [21] (Chapter III, Section 2, p. 102)) that for the $2 D$ Navier-Stokes equations there exists a finite dimensional global attractor $\mathcal{A}$ which is given by the omega limit set of a compact absorbing set in $H$. Thus, when $\epsilon$ tends to zero in (4) we obtain the deterministic Navier-Stokes equations. Questions concerning the existence and uniqueness of solutions for (4) have been studied extensively, see Bensoussan and Temam [2] for example. We are interested in the relationship between the random attractors $\mathcal{A}_{\epsilon}(\omega)$ associated to (4) for each $\epsilon \in(0,1]$ and the global attractor $\mathcal{A}$. Following the ideas in Crauel et al. [4] we are going to prove that there exists a random attractor for problem (4) and that, in fact, they are upper semicontinuous as $\epsilon$ goes to zero.

\subsection{Existence of random attractors}

For the study of this problem we introduce the change of variable

$$
v_{\epsilon}(t, \omega)=u_{\epsilon}(t, \omega)-\epsilon \phi z(t, \omega)
$$

where $z(t)$ denotes

$$
z(t)=\int_{-\infty}^{t} \exp (-\alpha(t-s)) d W(s)
$$

for $\alpha>0$ large enough and fixed. It is well known that $z(t)$ is a stationary process and its trajectories are $P-$ a.s. continuous.

It is clear that $v_{\epsilon}$ satisfies

$$
\frac{d v_{\epsilon}}{d t}+A v_{\epsilon}+B\left(v_{\epsilon}+\epsilon \phi z, v_{\epsilon}+\epsilon \phi z\right)=f+\alpha \epsilon \phi z-\epsilon A(\phi z) .
$$


Now equation (6) can be studied for each $\omega \in \Omega$, and so we know that there exists a unique solution for (6). Thus, we can define a random dynamical system associated to (4) given by

$$
\varphi_{\epsilon}(t, \omega) u_{0}=u_{\epsilon}(t, \omega)=v_{\epsilon}(t, \omega)+\epsilon \phi z(t, \omega) .
$$

Following the computations in Crauel et al. [4], taking care with the term containing the small parameter $\epsilon$, we can ensure the existence of a compact absorbing set $K_{\epsilon}(\omega)$ at time zero for each $\epsilon \in(0,1]$.

Indeed, taking the scalar product $\left(., v_{\epsilon}\right)$ in (6), and using (3), (5), (1) and the arguments in [4], we get

$\frac{1}{2} \frac{d}{d t}\left|v_{\epsilon}\right|^{2}+\left\|v_{\epsilon}\right\|^{2}+\left(B\left(v_{\epsilon}+\epsilon \phi z, v_{\epsilon}+\epsilon \phi z\right), v_{\epsilon}\right)=\left(f, v_{\epsilon}\right)+\alpha\left(\epsilon \phi z, v_{\epsilon}\right)-\left(\left(\epsilon \phi z, v_{\epsilon}\right)\right)$,

and

$$
\begin{aligned}
\left|v_{\epsilon}(t)\right|^{2} \leq & 2 c_{2}\left|u_{\epsilon}\left(t_{0}\right)\right|^{2} \exp \left(t_{0} \frac{\lambda_{1}}{8}\right)+2 c_{2} \epsilon^{2}|\phi|^{2}|z|^{2} \exp \left(t_{0}\left(\frac{\lambda_{1}}{8}\right)\right. \\
& +2 c_{2} \int_{t_{0}}^{0} g(\sigma) \exp \left(-\int_{\sigma}^{0}\left(\frac{\lambda_{1}}{4}-2 \epsilon c_{1}|z|\right) d \tau\right) d \sigma
\end{aligned}
$$

where

$$
g=2 c_{1} \epsilon|z|^{3}+\frac{2}{\lambda_{1}}|f|^{2}+\frac{2 \epsilon^{2} \alpha^{2}}{\lambda_{1}}|z|^{2}+\frac{1}{2} \epsilon|z|^{2},
$$

and $|z|$ denotes the modulus of $z$.

Then, there exists $t(\omega)$ (independent of $\epsilon$ ) such that for all $t_{0}<t(\omega)$ and $t \in[-1,0]$

$$
\left|v_{\epsilon}(t)\right|^{2} \leq r_{\epsilon}(\omega)
$$

with

$$
\begin{aligned}
r_{\epsilon}(\omega) & =1+2 c_{2} \int_{t_{0}}^{0} g(\sigma) \exp \left(-\int_{\sigma}^{0}\left(\frac{\lambda_{1}}{4}-2 \epsilon c_{1}|z|\right) d \tau\right) d \sigma \\
& +2 c_{2} \epsilon^{2}|\phi|^{2} \sup _{t_{0} \in(-\infty,-1]}\left|z\left(t_{0}\right)\right|^{2} \exp \left(t_{0} \frac{\lambda_{1}}{8}\right) .
\end{aligned}
$$

To derive an estimate in $V$, we take the scalar product by $v_{\epsilon}$ in $V$ in (6) making use of standard computations and obtain

$$
\begin{aligned}
\frac{1}{2} \frac{d}{d t}\left\|v_{\epsilon}\right\|^{2}+\left|A v_{\epsilon}\right|^{2}= & \left(\left(f, v_{\epsilon}\right)\right)+\alpha \epsilon\left(\left(\phi z, v_{\epsilon}\right)\right)-\epsilon\left(A \phi z, A v_{\epsilon}\right) \\
& -\left(B\left(v_{\epsilon}+\epsilon \phi z, v_{\epsilon}+\epsilon \phi z\right), A v_{\epsilon}\right),
\end{aligned}
$$

and

$$
\frac{d}{d t}\left\|v_{\epsilon}\right\|^{2} \leq C(t)+D(t)\left\|v_{\epsilon}\right\|^{2}
$$


where

$$
\begin{aligned}
C(t) & =4|f|^{2}+4 \alpha^{2} \epsilon^{2}|z|^{2}+4 \epsilon|A \phi z|^{2} \\
& +4 c_{3}^{2} \epsilon\left|v_{\epsilon}+\epsilon \phi z\right|\left|A \phi z\left\||| v_{\epsilon}+\epsilon \phi z\right\|^{2}\right. \\
& +32 c_{3}^{4} \epsilon\left|v_{\epsilon}+\epsilon \phi z\right|^{2}\|\phi z\|^{4} \\
& D(t)=32 c_{3}^{4} \epsilon\left|v_{\epsilon}+\epsilon \phi z\right|^{2}\left\|v_{\epsilon}\right\|^{2},
\end{aligned}
$$

and $c_{3}$ is the constant satisfying

$$
|B(u, u)| \leq c_{3}|u|^{1 / 2}|A u|^{1 / 2}\|u\| .
$$

Consequently, after integration in $[-1,0]$,

$$
\left\|v_{\epsilon}(0)\right\|^{2} \leq\left(\int_{-1}^{0}\left\|v_{\epsilon}(t)\right\|^{2} d t+\int_{-1}^{0} C(\sigma) d \sigma\right) \exp \int_{-1}^{0} D(\sigma) d \sigma
$$

and we finally obtain that there exists $\widehat{r}_{\epsilon}(\omega)$ such that, if $t_{0}<t(\omega)$,

$$
\left\|v_{\epsilon}(0)\right\|^{2} \leq \widehat{r}_{\epsilon}^{2}(\omega) .
$$

If we call $K_{\epsilon}(\omega)$ the ball in $V$ of radius $\widehat{r}_{\epsilon}(\omega)+\epsilon \phi\|z(0)\|$, we have a compact absorbing set (uniformly in $\epsilon$ ) in $H$ for $\varphi_{\epsilon}$. Furthermore, it is clear that

$$
\exists \lim _{\epsilon \searrow 0} \widehat{r}_{\epsilon}(\omega) \leq r_{d}
$$

with $r_{d}$ independent of $\omega \in \Omega$, and thus it is straightforward that the assumption (H2') of lemma 1 is satisfied. Thus for each $\epsilon>0$ there exists a random attractor $\mathcal{A}_{\epsilon}(\omega)$, and assumption (H2) of theorem 2 is guaranteed.

\subsection{Upper semicontinuity of attractors}

The following proposition gives (H1), and so the upper semicontinuity property:

Proposition 3 The solution $u_{\epsilon}\left(0, \omega ;-t_{0}, u_{0}\right)$ of (4) converges in $H P-$ a.s. as $\epsilon \rightarrow 0$ to the solution $u\left(t_{0} ; u_{0}\right)$ of the unperturbed problem, uniformly on bounded sets of initial conditions, that is, for P-almost every $\omega \in \Omega, t_{0} \in \mathbb{R}^{+}$ and $C \subset H$ bounded

$$
\lim _{\epsilon \backslash 0}\left|u_{\epsilon}\left(0, \omega ;-t_{0}, u_{0}\right)-u\left(t_{0} ; u_{0}\right)\right|=0 \quad \forall u_{0} \in C .
$$


Proof. Let $v_{\epsilon}(t, \omega)=u_{\epsilon}(t, \omega)-u(t)$ the difference between the solutions of the perturbed and the unperturbed equations with the same initial condition $u_{0}$ at $-t_{0}$. It is clear that $v_{\epsilon}$ satisfies

$$
\begin{aligned}
d v_{\epsilon}+A v_{\epsilon} d t+B\left(v_{\epsilon}+u, v_{\epsilon}+u\right)-B(u, u) d t & =\epsilon \phi d W(t) \\
v_{\epsilon}\left(-t_{0}\right) & =0
\end{aligned}
$$

As $B$ is bilinear, we can write this equation as

$$
d v_{\epsilon}+A v_{\epsilon} d t+\left(B\left(v_{\epsilon}, v_{\epsilon}\right)+B\left(v_{\epsilon}, u\right)+B\left(u, v_{\epsilon}\right)\right) d t=\epsilon \phi d W(t) .
$$

If we use now the change of variable

$$
z_{\epsilon}=v_{\epsilon}-\epsilon \phi W(t)
$$

we formally obtain

$$
\begin{aligned}
\frac{d z_{\epsilon}}{d t} & +A z_{\epsilon}+\epsilon A(\phi W(t))+B\left(z_{\epsilon}+\epsilon \phi W(t), z_{\epsilon}+\epsilon \phi W(t)\right. \\
& +B\left(z_{\epsilon}+\epsilon \phi W(t), u\right)+B\left(u, z_{\epsilon}+\epsilon \phi W(t)\right)=0
\end{aligned}
$$

Taking the scalar product with $z_{\epsilon}$, and by the bilinearity of $B$ and from (3), we obtain

$$
\begin{aligned}
\frac{1}{2} \frac{d}{d t}\left|z_{\epsilon}\right|^{2} & +\left(A z_{\epsilon}, z_{\epsilon}\right)+\left(\epsilon W(t) A \phi, z_{\epsilon}\right)+\left(B\left(z_{\epsilon}, \epsilon \phi W(t)\right), z_{\epsilon}\right) \\
& +\left(B(\epsilon \phi W(t), \epsilon \phi W(t)), z_{\epsilon}\right)+\left(B\left(z_{\epsilon}, u\right), z_{\epsilon}\right) \\
& +\left(B(\epsilon \phi W(t), u), z_{\epsilon}\right)+\left(B(u, \epsilon \phi W(t)), z_{\epsilon}\right)=0
\end{aligned}
$$

Using the properties of the operator $B$ and Young's inequality, the following estimates are straightforward

$$
\begin{aligned}
&\left(\epsilon W(t) A \phi, z_{\epsilon}\right) \leq \epsilon|W(t)||A \phi|\left|z_{\epsilon}\right| \leq \frac{\epsilon^{2} W(t)^{2}|A \phi|^{2}}{2}+\frac{\left|z_{\epsilon}\right|^{2}}{2} \\
&\left(B\left(z_{\epsilon}, \epsilon \phi W(t)\right), z_{\epsilon}\right) \leq \epsilon|W(t)|\left|\left(B\left(z_{\epsilon}, \phi\right), z_{\epsilon}\right)\right| \leq \beta \epsilon|W(t)|\left|z_{\epsilon}\right|^{2} \\
&\left(B(\epsilon \phi W(t), \epsilon \phi W(t)), z_{\epsilon}\right) \leq c_{3} \epsilon^{2} W(t)^{2}|\phi|^{1 / 2}|| \phi|||A \phi|^{1 / 2}\left|z_{\epsilon}\right| \\
& \leq \frac{c_{3}{ }^{2}}{2} \epsilon^{4} W(t)^{4}|\phi||| \phi \|^{2}|A \phi|+\frac{\left|z_{\epsilon}\right|^{2}}{2} \\
&\left(B\left(z_{\epsilon}, u\right), z_{\epsilon}\right) \leq c_{4}\left|z_{\epsilon}\right||| u|||| z_{\epsilon} \| \leq|| z_{\epsilon}||^{2}+c_{5}|| u||^{2}\left|z_{\epsilon}\right|^{2} \\
&\left(B(\epsilon \phi W(t), u), z_{\epsilon}\right) \leq \epsilon|W(t)|\left|\left(B(\phi, u), z_{\epsilon}\right)\right| \\
& \leq \epsilon|W(t)||\phi|^{1 / 2}|A \phi|^{1 / 2}|| u||\left|z_{\epsilon}\right| \\
& \leq \frac{1}{2} \epsilon^{2} W(t)^{2}|\phi||A \phi||| u \|^{2}+\frac{1}{2}\left|z_{\epsilon}\right|^{2}
\end{aligned}
$$




$$
\begin{aligned}
\left(B(u, \epsilon \phi W(t)), z_{\epsilon}\right) & \leq \epsilon|W(t)|\left|\left(B(u, \phi), z_{\epsilon}\right)\right| \\
& \leq \epsilon|W(t)||u|^{1 / 2}|| u||^{1 / 2} \| \phi||^{1 / 2}|A \phi|^{1 / 2}\left|z_{\epsilon}\right| \\
& \leq \epsilon|W(t)|^{2}|u||| u|||| \phi|||A \phi|+\frac{1}{2}\left|z_{\epsilon}\right|^{2} .
\end{aligned}
$$

¿From (9) and taking into account these inequalities we obtain

$$
\frac{d}{d t}\left|z_{\epsilon}\right|^{2} \leq h(t)+k(t)\left|z_{\epsilon}\right|^{2}
$$

where

$$
\begin{aligned}
h(t) & =K \epsilon\left(W(t)^{2}|A \phi|^{2}+W(t)^{4}|\phi|\|\phi\|^{2}|A \phi|\right. \\
& \left.+W(t)^{2}|\phi||A \phi|\|u\|^{2}+W(t)^{2}|u|\|u\|\|\phi\||A \phi|\right)
\end{aligned}
$$

and

$$
k(t)=2+\beta \epsilon|W(t)|+c_{5} \mid\|u\|^{2} .
$$

Then, by Gronwall's lemma,

$$
\left|z_{\epsilon}(t)\right|^{2} \leq h(t)+\int_{-t_{0}}^{t} h(s) k(s) \exp \left(\int_{s}^{t} k(\tau) d \tau\right) d s,
$$

and so $\left|z_{\epsilon}(t)\right|^{2} \rightarrow 0$ as $\epsilon \rightarrow 0$ for all $t \geq-t_{0}$. Then,

$$
\lim _{\epsilon \rightarrow 0}\left|v_{\epsilon}\right|^{2} \leq \lim _{\epsilon \rightarrow 0} 2\left(\left|z_{\epsilon}\right|^{2}+\left.\epsilon^{2}|\phi|^{2} W(t)\right|^{2}\right)=0,
$$

which finishes the proof by taking $t=0$.

Remark. In Flandoli and Langa [8] it is proved that the radius of the absorbing ball for the Navier-Stokes equations with additive noise has finite expectation, so that we can apply proposition 1 to this problem.

\section{Small random perturbation of a reaction- diffusion equation by additive white noise}

We now apply the results in section 2 to a reaction diffusion equation as it appears in Crauel and Flandoli [5]. As the ideas are similar to those in the last section, we will omit some of the computations. 


\subsection{Formulation of the problem}

Let $D \subset \mathbb{R}^{n}$ be an open bounded set with regular boundary and

$$
f(u)=\sum_{k=0}^{2 p-1} a_{k} u^{k}, \quad a_{2 p-1}<0 .
$$

We consider the following perturbation of a partial differential equation of reaction-diffusion type in $D$ by an additive white noise process multiplied by a small parameter $\epsilon$ :

$$
\left\{\begin{aligned}
d u_{\epsilon} & =\Delta u_{\epsilon} d t+f\left(u_{\epsilon}\right) d t+\epsilon \phi d W_{t} \quad \text { in } D \\
u_{\epsilon} & =0 \text { on } \partial D \\
u_{\epsilon}(0) & =u_{0}
\end{aligned}\right.
$$

where $W_{t}: \Omega \rightarrow \mathbb{R}, t \in \mathbb{R}$, is a one dimensional two-sided Wiener process.

Remarks. a) Some properties for these kind of problems, from the point of view of the theory of large deviations, can be found in Freidlin [9].

b) Again the analysis can be extended to the case $\epsilon \sum_{i=1}^{d} \phi^{i} d W_{t}^{i}$.

To pose this problem into a variational form we introduce the following spaces: $H=L^{2}(D)$ (with $(.,),.|$.$| its scalar product and norm respectively),$ $V=H_{0}^{1}(D)(((.,)),.\|\|),. Z=L^{2 p}(D)$ and $Z^{\prime}=L^{(2 p)^{\prime}}(D)$, with $(2 p)^{\prime}=$ $(2 p-1) / 2 p$. It is known that $(10)$ can be expressed as a differential equation in $H$ :

$$
\left\{\begin{aligned}
d u_{\epsilon} & =A u_{\epsilon} d t+F\left(u_{\epsilon}\right) d t+\epsilon \phi d W_{t} \quad \text { in } H \\
u_{\epsilon}(0) & =u_{0}
\end{aligned}\right.
$$

where $A: D(A) \subset H \rightarrow H, A u=\Delta u, F: Z \rightarrow Z^{\prime}$ and we take $\phi \in D(A)$.

Under these conditions, results for the existence of a global attractor $\mathcal{A}$ for the unperturbed systems are known (see, for instance, Temam [21] (Chapter III, p.81)).

We study (11) by the change of variable

$$
v_{\epsilon}(t, \omega)=u_{\epsilon}(t, \omega)-\epsilon \phi W_{t}(\omega) .
$$

It is easy to check that $v_{\epsilon}(t, \omega)$ satisfies

$$
d v_{\epsilon}=A v_{\epsilon} d t+F\left(v_{\epsilon}+\epsilon \phi W_{t}\right) d t+\epsilon A\left(\phi W_{t}\right) .
$$

Equation (12) can be studied for each $\omega \in \Omega$, so that applying the results for the existence and uniqueness of solutions that appear in Temam [21] (Chapter III, theorem 1.1), we obtain that $P$-almost every $\omega \in \Omega$, the following holds 
i) for all $t_{0}<T$ and all $v_{0} \in H$ there exists a unique solution of (12), with $v_{\epsilon}\left(t_{0}\right)=v_{0}, v_{\epsilon} \in C\left(\left[t_{0}, T\right] ; H\right) \cap L^{2}\left(t_{0}, T ; V\right) \cap L^{2 p}\left(\left[t_{0}, T\right] ; Z\right)$,

ii) if $v_{0} \in V$, the solution belongs to $C\left(\left[t_{0}, \infty\right) ; V\right) \cap L_{l o c}^{2}\left(t_{0}, \infty ; D(A)\right)$,

iii) denoting such a solution by $v_{\epsilon}\left(t, \omega ; t_{0}, v_{0}\right)$, the mapping $v_{0} \mapsto v_{\epsilon}\left(t, \omega ; t_{0}, v_{0}\right)$ is continuous for all $t \geq 0$.

¿From the mapping $v_{0} \mapsto v_{\epsilon}\left(t, \omega ; t_{0}, v_{0}\right)$ we can define a stochastic flow $\varphi_{\epsilon}(t, \omega) u_{0}=$ $v_{\epsilon}\left(t, \omega ; 0, u_{0}\right)+\epsilon \phi W_{t}$. This is the stochastic flow associated with (11).

We will show that the hypotheses of theorem 2 are satisfied:

\subsection{Existence of random attractors}

In this section we summarize the results from Crauel and Flandoli [5], section 5 , which lead to the existence of a compact random attractor for each $\varphi_{\epsilon}$ (theorem 5.6 in their paper). We omit the calculations as they are similar to those made in [5], and to those of the previous section; as there, the main change is that we have to be careful to keep track of the small parameter $\epsilon$ which multiplies the random terms in the equations. Taking this into account the following results are straightforward:

Lemma 3 a) For all $u_{\epsilon} \in D(A) \cap Z$ we have $P$-a.s.

$$
-\left(A\left(u_{\epsilon}-\epsilon \phi W_{t}\right), F\left(u_{\epsilon}\right)\right) \leq \beta\left\|u_{\epsilon}\right\|^{2}+\gamma\left\|u_{\epsilon}\right\|_{Z}^{2 p}+\epsilon p_{1}(t, \omega)
$$

where $\beta, \gamma>0$ and

$$
p_{1}(t, \omega)=c_{4}|| A \phi\left\|_{Z^{\prime}}^{2 p}\left|W_{t}\right|^{2 p}+c_{3}|| A \phi\right\|_{Z^{\prime}}\left|W_{t}\right|, \quad c_{i}>0 .
$$

b) For all $u_{\epsilon} \in Z$ we have $P-$ a.s.

$$
\left(u_{\epsilon}-\epsilon \phi W_{t}, F\left(u_{\epsilon}\right)\right) \leq-\delta_{0}\left\|u_{\epsilon}\right\|_{Z}^{2 p}+c_{5}|D|+\epsilon p_{2}(t, \omega),
$$

where $\delta_{0}, c_{5}>0$ and

$$
p_{2}(t, \omega)=c_{6}|| \phi\left\|_{Z^{\prime}}^{2 p}\left|W_{t}\right|^{2 p}+c_{3}|| \phi\left|\|_{Z^{\prime}}\right| W_{t} \mid, \quad c_{i}>0 .\right.
$$


Remark. Note that the functions $p_{i}(t, \omega)$ are nonnegative and have, at most, polynomial growth as $t \rightarrow-\infty$ for $P$ - almost every $\omega \in \Omega$.

Proposition 4 (absorption in $H$ at $t=-1$ )

There exists a random radius $r_{\epsilon}(\omega)>0$ such that for all $\rho>0$ there exists $t(\rho) \leq-1$ (independent of $\epsilon$ ) such that the following holds P-a.s.: for all $t_{0} \leq t(\rho)$ and all $u_{0} \in H$ with $\left|u_{0}\right| \leq \rho$, the solution of (12) verifies

$$
\left|v_{\epsilon}\left(-1, \omega ; t_{0}, u_{0}-\epsilon \phi W_{t_{0}}(\omega)\right)\right|^{2} \leq r_{\epsilon}^{2}(\omega) .
$$

Lemma 4 There exists a random variable $c_{1}(\epsilon, \omega)$ such that $\forall \rho>0$ there exists $t(\rho)<-1$ (independent of $\epsilon$ ) such that the following holds $P$-a.s.: for all $t_{0} \leq t(\rho)$ and all $u_{0} \in H$ with $\left|u_{0}\right| \leq \rho$, we have

a)

$$
\int_{-1}^{0}\left\|v_{\epsilon}(s, \omega)\right\|^{2} d s \leq 2 c_{1}(\epsilon, \omega)
$$

b)

$$
\int_{-1}^{0}\left\|u_{\epsilon}(s, \omega)\right\|_{Z}^{2 p} d s \leq \frac{1}{2 \delta_{0}} c_{1}(\epsilon, \omega)
$$

Proposition 5 (absorption in $V$ at $t=0$ )

There exists a random radius $\widetilde{r}_{\epsilon}(\omega)$ such that for all $\rho>0$ there exists $\widetilde{t}(\rho) \leq$ -1 (independent of $\epsilon$ ) such that the following holds $P-$ a.s.: for all $t_{0} \leq \widetilde{t}(\rho)$ and all $u_{0} \in H$ with $\left|u_{0}\right| \leq \rho$ denote by $u_{\epsilon}\left(t, \omega ; t_{0}, u_{0}\right)$ the solution of (10) on $\left[t_{0},+\infty\right)$, then

$$
\left\|u_{\epsilon}\left(0, \omega ; t_{0}, u_{0}\right)\right\|^{2} \leq \widetilde{r}_{\epsilon}^{2}(\omega),
$$

with

$$
\begin{aligned}
\widetilde{r}_{\epsilon}(\omega)^{2}= & k_{2} r_{d}^{2}+\sup _{t_{0} \leq-1} \epsilon g_{1}\left(\left|W\left(t_{0}, \omega\right)\right|\right) e^{\frac{\lambda_{1}}{2}}+\epsilon \int_{-\infty}^{-1} e^{\frac{\lambda_{1} s}{2}} g_{2}(|W(s, \omega)| d s \\
& +\epsilon \int_{-1}^{0} e^{\frac{\lambda_{1} s}{2}} g_{3}(|W(s, \omega)| d s,
\end{aligned}
$$


$g_{i} \geq 0, i=1,2,3$, are polynomials whose coefficients depend on the norm of the function $\phi$ on $H^{2}(D)$, on $f$ and the domain $D$, and $r_{d}^{2}=2+\frac{4|D| c_{5}}{\lambda_{1}}$, $k_{2}=k_{2}\left(\beta, \gamma, \delta_{0}\right)($ see $[5])$.

These results lead to the following theorem about the existence of random attractors for $\varphi_{\epsilon}$.

Theorem 3 The stochastic flow $\varphi_{\epsilon}$ associated with the reaction-diffusion equation has a compact random attractor denoted by $\mathcal{A}_{\epsilon}(\omega)$.

Furthermore, as the shift $\theta_{t}: \Omega \rightarrow \Omega$ is ergodic, for each $\epsilon>0$ there exists a bounded set $K_{\epsilon} \subset H$ such that $P-$ a.s.

$$
\mathcal{A}_{\epsilon}(\omega)=\Lambda_{\epsilon}\left(K_{\epsilon}, \omega\right)
$$

Remark. For the second part of the result see Crauel et al. [4], page 11.

¿From proposition 5, and taking into account the polynomial growth of the functions $g_{i}, i=1,2,3$, and the obvious growth in $\epsilon$ of $\widetilde{r}_{\epsilon}(\omega),\left(\mathrm{H} 2^{\prime}\right)$ of lemma 1 is straightforward, and hence $(\mathrm{H} 2)$ of theorem 2 is assured. Furthermore, it is easy to prove that the radius $\widetilde{r}_{\epsilon}(\omega)$ has finite expectation for all $p \geq 1$, so that the conditions of proposition 1 are satisfied and the last characterization in theorem 3 can be improved as we can ensure that there exists a ball $B_{R} \subset H$ such that, for all $\epsilon \in(0,1]$

$$
\mathcal{A}_{\epsilon}(\omega)=\Lambda_{\epsilon}\left(B_{R}, \omega\right) \quad \text { with probability one. }
$$

The following lemma gives (H1):

Lemma 5 The solution $u_{\epsilon}\left(0, \omega ;-t_{0}, u_{0}\right)$ of (11) converges in $H$ as $\epsilon \rightarrow 0$ to the solution $u\left(t_{0} ; u_{0}\right)$ of the unperturbed problem, uniformly on bounded sets of initial conditions, that is, for $P$-almost every $\omega \in \Omega, t_{0} \in \mathbb{R}^{+}$and $B \subset H$ bounded, we have that

$$
\lim _{\epsilon \searrow 0}\left|u_{\epsilon}\left(0, \omega ;-t_{0}, u_{0}\right)-u\left(t_{0} ; u_{0}\right)\right|=0 \quad \forall u_{0} \in B
$$


Proof. Let $v_{\epsilon}(t)=u_{\epsilon}(t)-u(t)$ the difference between the solutions of the perturbed and the unperturbed systems. Then it is clear that $v_{\epsilon}$ satisfies

$$
d v_{\epsilon}=A v_{\epsilon} d t+\left(F\left(u_{\epsilon}\right)-F(u)\right) d t+\epsilon \phi d W(t), \quad v_{\epsilon}\left(-t_{0}\right)=0 .
$$

If we use again the change of variable

$$
z_{\epsilon}=v_{\epsilon}-\epsilon \phi W(t)
$$

we get that $z_{\epsilon}$ satisfies

$$
\frac{d}{d t} z_{\epsilon}=A z_{\epsilon}+\epsilon A(\phi W(t))+F\left(u_{\epsilon}\right)-F(u)
$$

and so, taking scalar product in $H$ with $z_{\epsilon}$ we obtain

$$
\begin{aligned}
\frac{d}{d t}\left|z_{\epsilon}\right|^{2} & =-|| z_{\epsilon} \|^{2}+\left(\epsilon W(t) A \phi, z_{\epsilon}\right)+\left(F\left(u_{\epsilon}\right)-F(u), u_{\epsilon}-u-\epsilon \phi W(t)\right) \\
& \leq-\left.|| z_{\epsilon}\right|^{2}+\frac{\epsilon}{2}|W(t)|^{2}|A \phi|^{2}+\frac{1}{2}\left|z_{\epsilon}\right|^{2}+\left(F\left(u_{\epsilon}\right)-F(u), u_{\epsilon}-u\right) \\
& -\left(F\left(u_{\epsilon}\right)-F(u), \epsilon \phi W(t)\right) \\
& \leq-|| z_{\epsilon} \|^{2}+\frac{\epsilon}{2}|W(t)|^{2}|A \phi|^{2}+\frac{1}{2}\left|z_{\epsilon}\right|^{2}+k\left|u_{\epsilon}-u\right|^{2} \\
& +\epsilon|\phi||W(t)|\left|F\left(u_{\epsilon}\right)-F(u)\right|
\end{aligned}
$$

where we have used that $\forall x, y \in \mathbb{R}$

$$
(F(x)-F(y))(x-y) \leq k|x-y|^{2},
$$

and clearly

$$
\left|u_{\epsilon}-u\right|^{2} \leq k_{1}\left|z_{\epsilon}\right|^{2}+k_{2}|2 \epsilon \phi W(t)|
$$

Thus, we finally get

$$
\frac{d}{d t}\left|z_{\epsilon}\right|^{2} \leq h(t)+g\left|z_{\epsilon}\right|^{2}
$$

and so, as in the previous section and after using Gronwall's lemma, we have that $P-$ a.s.

$$
\lim _{\epsilon \rightarrow 0}\left|z_{\epsilon}(t)\right|^{2}=0, \quad \forall t \geq-t_{0}
$$

and then

$$
\lim _{\epsilon \rightarrow 0}\left|v_{\epsilon}(t)\right|^{2}=\lim _{\epsilon \rightarrow 0}\left|u_{\epsilon}(t)-u(t)\right|^{2}=0
$$

which finishes the proof by taking $t=0$.

Thus, as the conditions of theorem 2 are fulfilled we have the upper semicontinuity property for the random attractors $\mathcal{A}_{\epsilon}(\omega)$ as $\epsilon \rightarrow 0$. 


\section{Conclusion}

We have proved a general result on the upper semicontinuity of attractors for small random perturbations of dynamical systems and shown that it can be applied to some problems where the existence of random attractors has already been proved. We think that the result should also be applicable to similar problems. As indicated in the introduction, there are other possible definitions for the attractor of a stochastic differential equation. The result we have proved shows that the definition of random attractor seems to be a good generalisation of the classical concept of global attractor, as the random attractor, which, in general, is not uniformly bounded in $\omega \in \Omega$, converges (in the sense of upper semicontinuity) to the deterministic global attractor.

As in the deterministic case, the lower semicontinuity property is not true in general. Indeed, an example is given in Crauel and Flandoli [6] for which the random attractor converges towards a subset of the deterministic point attractor $\left(\hat{\mathcal{A}}=\cup_{x \in X} \Lambda(x)\right.$, see Ladyzhenskaya [15]). We conjecture that this result is true in general, since small random perturbations should remove the unstable orbits which lie in the standard deterministic global attractor $\mathcal{A}$.

\section{ACKNOWLEDGEMENTS}

The authors wish to thank the referee for his/her helpful and detailed suggestions.

The first two authors want to thank Prof. José Real for his helpful comments and valuable advice on the topic of this paper. James C. Robinson would like to thank Enrique Fernández Cara and the EDAN department for the invitation to visit the department in Seville and all there for their kindness and hospitality.

This work has been partially supported by D.G.I.C.Y.T. (Spain) proyecto No. PB93-1196. 


\section{References}

[1] L. Arnold and H. Crauel, Random dynamical systems, Lyapunov Exponents, Proceedings, Oberwolfach, Lecture Notes in Mathematics 1486 (1990), 1-22.

[2] A. Bensoussan and R. Temam, Equations stochastiques du type NavierStokes, J. Funct. Anal. 13 (1973), 195-222.

[3] H. Crauel, Invariant measures are supported by random attractors, preprint (1996).

[4] H. Crauel, A. Debussche and F. Flandoli, Random attractors, prepublication 95-27, Université de Paris-Sud (1995).

[5] H. Crauel and F. Flandoli, Attractors for random dynamical systems, Probability Theory and Related Fields 100 (1994), 365-393.

[6] H. Crauel and F. Flandoli, Additive noise destroys a pitchfork bifurcation, preprint (1996).

[7] C.R. Doering and J.D. Gibbon, Applied Analysis of the Navier-Stokes Equations, Cambridge University Press, Cambridge (1995).

[8] F. Flandoli and J.A. Langa, Determining modes for dissipative random dynamical systems, to appear in Stochastics and Stochastics Reports.

[9] M.I. Freidlin, Semilinear PDE's and limit theorems for large deviations, Ecole d'Eté de Probabilités de Saint-Flour XX-1990 LNM, 1527 (1992), 1-108 Berlin Heidelberg: Springer.

[10] J. Hale, Asymptotic behavior of dissipative systems, Mathematical Surveys and Monographs, AMS, Providence (1988).

[11] J. Hale, X. Lin and G. Raugel, Upper semicontinuity of attractors for approximations of semigroups and PDE's, Math. Comp., 50 (1988), number 181, 89-123.

[12] J. Hale and G. Raugel, Upper semicontinuity of the attractor for a singularly perturbed hyperbolic equation, J. Differ. Equations, 73 (1988), 197214 
[13] A. Haraux, Attractors of asymptotically compact processes and applications to nonlinear partial differential equations, Commun. Part. Diff. Eq. 13 (11) (1988), 1383-1414.

[14] P.E. Kloeden and D.J. Stoiner, Cocycle attractors in nonautonomously perturbed differential equations, CADSEM Report 96-010, Deakin University (1996).

[15] O. Ladyzhenskaya, Attractors for semigroups and Evolution Equations, Accademia Nazionale dei Lincei, Cambridge University Press (1991).

[16] H. Morimoto, Attractors of probability measures for semilinear stochastic evolution equations, Stochastic Analysis and Applications 10 (2) (1992), 205-212.

[17] B. Øksendal, Stochastic Differential Equations, Springer-Verlag, New York (1992).

[18] K. Schmalfuß, Backward cocycles and attractors and attractors of stochastic differential equations, in V. Reitmann, T. Redrich, and N. Kosch (eds.), International Seminar on Applied Mathematics-Nonlinear Dynamics: Attractor Approximation and Global Behaviour (1992), 185-192.

[19] K. Schmalfuß, Long-time behaviour of the stochastic Navier-Stokes equation, Math. Nachr. 152 (1991), 7-20.

[20] G.R. Sell, Non-autonomous differential equations and topological dynamics I, II, Trans. Amer. Math. Soc. 127 (1967), 241-262, 263-283.

[21] R. Temam, Infinite-Dimensional Dynamical Systems in Mechanics and Physics, Springer-Verlag, New York (1988).

[22] M.I. Vishik, Asymptotic behaviour of solutions of evolutionary equations, Cambridge University Press (1992). 